\title{
Realization of finite Abelian groups by nets in $\mathbb{P}^{2}$
}

\author{
Sergey Yuzvinsky
}

\begin{abstract}
In this paper, we study special configurations of lines and points in the complex projective plane, so called $k$-nets. We describe the role of these configurations in studies of cohomology on arrangement complements. Our most general result is the restriction on $k$ : it can equal only 3,4 , or 5 . The most interesting class of nets is formed by 3 -nets that relate to finite geometries, latin squares, loops, etc. All known examples of 3-nets in $\mathbb{P}^{2}$ realize finite Abelian groups. We study the question of which groups can be realized in this way. Our main result is that, except for groups with all invariant factors below 10, the realizable groups are isomorphic to subgroups of a 2-torus. This follows from the 'algebraization' result asserting that, in the dual plane, the points dual to lines of a net lie on a plane cubic.
\end{abstract}

\section{Introduction}

The notion of a net, that is, a discrete analogue of a web, has a long history related to discrete geometries, latin squares, loops, etc. (e.g. see [DK74]). It turned out recently that nets play a special role in the study of the cohomology of local systems on the complements of complex line arrangements (see $\S 2$ ). Since the latter discovery attracted the author to nets, we do not even define abstract nets in this paper but from the beginning restrict our considerations to nets in the complex projective plane $\mathbb{P}^{2}$. These nets can also be viewed as complex representations of certain matroids of rank 3 .

Definition 1.1. Let $k$ be an integer, $k \geqslant 3$. A $k$-net in $\mathbb{P}^{2}$ is a pair $(\mathcal{A}, \mathcal{X})$ where $\mathcal{A}$ is a finite set of lines partitioned into $k$ subsets $\mathcal{A}=\bigcup_{i=1}^{k} \mathcal{A}_{i}$ and $\mathcal{X}$ is a finite set of points subject to the following conditions:

i) for every $i \neq j$ and every $l \in \mathcal{A}_{i}, l^{\prime} \in \mathcal{A}_{j}$, we have $l \cap l^{\prime} \in \mathcal{X}$;

ii) for every $X \in \mathcal{X}$ and every $i(i=1,2, \ldots, k)$ there exists a unique $l \in \mathcal{A}_{i}$ passing through $X$.

The definition of a $k$-net implies immediately that $\left|\mathcal{A}_{i}\right|=|l \cap \mathcal{X}|$ for every $i, 1 \leqslant i \leqslant k$, and every $l \in \mathcal{A}$. This number, which we denote by $m$, is called the order of the net. We will often use the term $(k, m)$-net for a $k$-net of order $m$. For a $(k, m)$-net we have $|\mathcal{X}|=m^{2}$. We will always consider nontrivial nets defined by the condition $m>1$.

Notice that we do not put any restrictions on the combinatorics of lines inside a class; they can form an arbitrary arrangement from general position one to a pencil.

Our first result about nets (Theorem 3.2) asserts that the only possible values for $k$ are 3, 4, and 5 (cf. [LY00]). By putting extra conditions on combinatorics inside classes, one can restrict $k$ even more. For instance, if two classes are pencils, then $k=3$.

Received 16 May 2003, accepted in final form 28 September 2003, published online 15 October 2004. 2000 Mathematics Subject Classification 52C30, 05B30 (primary), 14H50 (secondary).

Keywords: nets, loops, complex projective plane.

This journal is (c) Foundation Compositio Mathematica 2004. 


\section{Realization of finite Abelian groups by nets in $\mathbb{P}^{2}$}

Since 3-nets form the most interesting class of nets and only one example of other nets in $\mathbb{P}^{2}$ is known to the author, we restrict our considerations to $k=3$. In this case, there is the pairing $\mathcal{A}_{1} \times \mathcal{A}_{2} \rightarrow \mathcal{A}_{3}$ defined by assigning to $l_{i} \in \mathcal{A}_{i}(i=1,2)$ the line from $\mathcal{A}_{3}$ passing through $l_{1} \cap l_{2}$. After any identification of each class with a set $H$, this pairing defines a binary operation on $H$ converting $H$ into a quasigroup. A general theorem of quasigroup theory (e.g. see [DK74, Theorem 8.1.4]) asserts that one can always find identifications such that $H$ is a loop, that is, all group axioms hold for it except perhaps for associativity. If this loop is a group, we say that the net realizes the group.

There are conditions on a net in $\mathbb{P}^{2}$ implying that it represents a particular group. For instance, if all classes of a $(3, m)$-net are pencils, then the net realizes $\mathbb{Z}_{m}$ (Proposition 3.3). No example of a 3 -net in $\mathbb{P}^{2}$ is known to the author where the net does not realize an Abelian group. The main part of this paper deals with the question of which Abelian groups are realizable by nets in $\mathbb{P}^{2}$.

Our main result in this direction is somewhat similar to the theorem of H. Graf and R. Sauer in 1924 about algebraization of webs. Following the theory of webs, we say that a 3-net is algebraic if there exists a cubic curve in the dual plane $\mathbb{P}^{*}$ passing through all the points dual to the lines of the net. We prove (Theorems 5.3 and 5.4) that nets of a wide class are algebraic. This class contains the nets realizing arbitrary cyclic groups and also Abelian groups with elements of order larger than 9 .

Using the algebraization result, we can prove our negative results about realization. The main statement (Corollary 5.7) asserts that a finite Abelian group $H$ with an element of order larger than 9 can be realized if and only if it is the direct sum of not more than two cyclic groups. The 'if' part is true without any restriction on the orders of elements (Theorem 4.4). If $H$ has an element of order larger than 6 , then it can be realized by a net with one class being a pencil if and only if $H$ is cyclic. Also any cyclic group can be realized with all three classes being pencils and this realization is projectively unique (Proposition 3.3).

We conjecture that the realization result holds without any condition on the order of the elements. Also we suggest problems of finding more examples of 4- and 5-nets in $\mathbb{P}^{2}$ and 3-nets not realizing Abelian groups (or of proving that such examples do not exist).

The paper is organized as follows. Section 2 is devoted completely to motivations and is not needed to understand the rest of this paper. In $\S 3$, we prove general restrictions on $k$ for a $k$-net in $\mathbb{P}^{2}$. In particular, the complete classification of nets whose three classes are pencils is given. In $\S 4$ (and for the rest of this paper), we restrict our considerations to 3-nets and study which Abelian groups can be realized by them. We exhibit the first positive and negative examples of realization. In $\S 5$, we prove our main theorems about the property of nets being algebraic and about Abelian group realization. For that we need first to study the operations defined by the collinearity of points on reducible cubics. Finally in $\S 6$, we state conjectures and unsolved problems.

\section{Motivation: essential components of characteristic varieties}

As in the Introduction, we denote by $\mathcal{A}$ a finite set $\left\{l_{1}, \ldots, l_{n}\right\}$ of lines in the complex projective plane $\mathbb{P}^{2}$ and put $M=\mathbb{P}^{2} \backslash \bigcup_{i=1}^{n} l_{i}$. The algebra $H^{*}(M, \mathbb{C})$ is naturally isomorphic to the Orlik-Solomon algebra $A$ of $\mathcal{A}$. The latter algebra is generated by skew-commutative degree- 1 generators $e_{1}, \ldots, e_{n}$ satisfying certain extra relations of degree at least 2 (see [OT92]). Computing the cohomology of local systems on $M$ is a much more subtle task. Every rank-one local system on $M$ is defined by a character $H_{1}(M) \rightarrow \mathbb{C}^{*}$ whence the set of all those systems form the $n$-dimensional torus $T=H^{1}\left(M, \mathbb{C}^{*}\right)$. For every $k \geqslant 1$ we put $\Sigma_{k}=\left\{\mathcal{L} \in H^{1}\left(M, \mathbb{C}^{*}\right) \mid \operatorname{dim} H^{1}(M, \mathcal{L}) \geqslant k\right\}$. This is an algebraic subvariety of the torus called the kth characteristic variety of $M$ (or of $\mathcal{A}$ ).

Here we will consider only the irreducible components of $\Sigma_{k}$ containing the identity $\mathbf{1}=$ $(1,1, \ldots, 1)$ of the torus, i.e. the constant local system. For such a component, it is possible to 


\section{S. YUZVINSKY}

describe its tangent space at $\mathbf{1}$. Note that the multiplication by an element $a \in A_{1}$ defines a differential on $A$ of degree 1 whence the cohomology spaces $H^{p}(A, a)$. This allows one to define resonance subvarieties of $A_{1}$ via $R_{k}=\left\{a \in A_{1} \mid \operatorname{dim} H^{1}(A, a) \geqslant k\right\}$. Now since the above generators of $A$ form a basis of $A_{1}$ we can identify $A_{1}$ with the tangent space to $T$ at $\mathbf{1}$ (i.e. the Lie algebra of $T$ ). The following result is just a little stronger than the results proved in [LY00].

TheOREM 2.1. For every component $\mathcal{V}$ of $\Sigma_{k}$ containing $\mathbf{1}$, the tangent space of $\mathcal{V}$ is a component of $R_{k}$.

Now let us summarize results from [LY00] (discussed in more detail in [YO01, ch. 7]). Every irreducible component of $R_{k}$ is defined by a subarrangement $\mathcal{B} \subset \mathcal{A}$ and a set $\mathcal{X}$ of multiple points of $\mathcal{B}$. If $J$ is the incidence matrix of the pair $(\mathcal{X}, \mathcal{B})$ and $E$ is the $|\mathcal{B}| \times|\mathcal{B}|$ matrix whose entries all equal 1, then the matrix $Q=J^{\mathrm{t}} J-E$ should have at least $k+2$ nondecomposable components of affine type (see [Kac90]) that cover every $X \in \mathcal{X}$ and $\mathcal{B}$ should be maximal with respect to this property. In the rest of this paper we will consider only essential components for which $\mathcal{B}=\mathcal{A}$.

One of the corollaries of the description of a component of $R_{k}$ is that the multiplicity of every point from $\mathcal{X}$ is at least $k+2$ (see [LY00, Proposition 2.5]). Thus the most 'economical' components are those for which all points of $\mathcal{X}$ have the same multiplicity equal to $k+2$. These components correspond to the full graphs in the sense of $[L Y 00, \S 6]$, and the combinatorics of them has been described in [YO01]. In terms of nets in $\mathbb{P}^{2}$ (Definition 1.1) the latter result just asserts that those components correspond to the case where the pair $(\mathcal{A}, \mathcal{X})$ is a $(k+2)$-net.

Given a $(k+2)$-net $(\mathcal{A}, \mathcal{X})$ in $\mathbb{P}^{2}$ it is easy to write explicitly the respective essential component $V=V(\mathcal{X})$ of $R_{k}$ for $\mathcal{A}$. The partition $\mathcal{A}=\bigcup_{i=1}^{k+2} \mathcal{A}_{i}$ induces the respective partition of the basis $\left(e_{1}, e_{2}, \ldots, e_{n}\right)$ of $A_{1}$. Then $V$ consists of all $a=\left(x_{1}, \ldots, x_{n}\right) \in A_{1}$ such that $\sum_{i=1}^{n} x_{i}=0$ and $x_{i}=x_{j}$ on each block of the partition.

A subset $\mathcal{B} \subset \mathcal{A}$ formed by a pencil of lines also defines a component of $R_{k}, k=|\mathcal{B}|-2$. This component is not essential unless $\mathcal{B}=\mathcal{A}$ and is called local. The local component corresponds to the trivial $(k, 1)$-net formed by $\mathcal{B}$ and the base of the pencil.

Example 2.2. The arrangement of lines in $\mathbb{P}^{2}$ given by the linear functionals $(x, y, z, x-y, x-z, y-z)$ can be partitioned into three parts, $\{x, y-z\},\{y, x-z\}$, and $\{z, x-y\}$. It is easy to check that this partition defines a $(3,2)$-net with all four triple points of the intersections. The respective component of $R_{1}$ is the unique nonlocal (essential) component.

\section{Restrictions on $k$-nets in $\mathbb{P}^{2}$}

Unlike the theory of the abstract $(k, m)$-nets (see [DK74]) where there are no general restrictions on $k$ and $m$, one can obtain quite a few restrictions on these parameters for nets in $\mathbb{P}^{2}$.

First we observe that a $(k, m)$-net induces a pencil of curves of degree $m$ with $k$ very special fibers. (In [LY00] these pencils were used in a more general situation using deep results by Arapura; here we introduce them elementarily.)

For a $(k, m)$-net $(\mathcal{A}, \mathcal{X})$ with a partition $\mathcal{A}=\bigcup_{i=1}^{k} \mathcal{A}_{i}$, consider $k$ split curves of degree $m$ given by $C_{i}=\bigcup_{l \in \mathcal{A}_{i}} l, 1 \leqslant i \leqslant k$. The defining ideal of $C_{i}$ is generated by the polynomial $g_{i}=\prod_{j} \alpha_{i j}$ where $\left\{\alpha_{i j} \mid j=1, \ldots, m\right\}$ are some fixed linear functionals whose kernels run through the lines from $A_{i}$.

The following lemma is a simple case of Max Noether's $A F+B G$ Theorem.

Lemma 3.1. All curves $C_{i}$ are fibers of the pencil generated by any two of them.

Proof. Choose two of the curves, say $C_{1}$ and $C_{2}$. Since the curves intersect at $m^{2}$ (simple) points, the ideal $I$ generated by $g_{1}$ and $g_{2}$ in the polynomial ring is radical. Also any other curve $C_{i}$ passes 


\section{Realization of finite Abelian groups by nets in $\mathbb{P}^{2}$}

through all these intersection points. By the Nullstellensatz we have $g_{i} \in I$. Since the degree of $g_{i}$ is again $m$, we obtain the result.

Lemma 3.1 implies that there is a rational map $\phi: \mathbb{P}^{2} \rightarrow \mathbb{P}^{1}$ with poles at the points of the net that sends $p \in \mathbb{P}^{2}$ to the point $(c: d) \in \mathbb{P}^{1}$ such that $c g_{1}(p)+d g_{2}(p)=0$. Blowing up all the points of the net we obtain a variety $P^{\prime}$ and a regular map $\psi: P^{\prime} \rightarrow \mathbb{P}^{1}$ whose preimages of points are the proper transforms of fibers of the pencil. This allows us to prove the following properties of the net (cf. [LY00, Proposition 7.3]).

\section{THEOREM 3.2.}

i) For an arbitrary $(k, m)$-net in $\mathbb{P}^{2}$, the only possible values for $(k, m)$ are: $(k=3, m \geqslant 2)$, $(k=4, m \geqslant 3)$ and $(k=5, m \geqslant 6)$.

ii) If one class of the net is a pencil, then the only possible values are: $(k=3, m \geqslant 2)$ and $(k=4, m \geqslant 4)$.

iii) If two classes are pencils, then the only possible value for $k$ is 3 .

Proof. To cover all the cases, let us assume that $r$ classes of the net are pencils, $0 \leqslant r \leqslant k$. We estimate the Euler characteristic $\chi\left(P^{\prime}\right)$. Since we got $P^{\prime}$ by blowing up $m^{2}$ points of $\mathbb{P}^{2}$, we have $\chi\left(P^{\prime}\right)=3+m^{2}$. On the other hand, let us estimate the Euler characteristics $\chi$ of fibers of $\psi$. The general position fiber is a smooth curve of degree $m$ whence $\chi=3 m-m^{2}$. At least $k$ fibers are unions of $m$ lines, whence for each of these fibers $m(5-m) / 2 \leqslant \chi \leqslant m+1$. Here the left-hand side value is reached by generic configurations of lines and the right-hand side value by a pencil of lines. Combining this information we have

$$
3+m^{2} \geqslant(2-k)\left(3 m-m^{2}\right)+r(m+1)+(k-r) \frac{m(5-m)}{2}
$$

or equivalently (since $m>1$ )

$$
k \leqslant 6 \frac{m-1}{m}-r \frac{m-2}{m} .
$$

Ignoring the second summand we immediately get the first statement.

If $r=1$ the inequality (3.2) becomes

$$
k \leqslant 5-\frac{4}{m}
$$

which implies the second statement. Finally if $r=2$ we have

$$
k \leqslant 4-\frac{2}{m}
$$

which implies the last statement.

It turns out that nets whose classes are all pencils can be classified completely up to projective isomorphism.

Proposition 3.3. For any $(3, m)$-net $N=(\mathcal{A}, \mathcal{X})$ in $\mathbb{P}^{2}$ whose classes are all pencils there exists a homogeneous coordinate system such that the classes $\mathcal{A}_{i}$ of $N$ are given by the equations $x^{m}-y^{m}=0, x^{m}-z^{m}=0$ and $y^{m}-z^{m}=0$ respectively. In particular $N$ realizes $\mathbb{Z}_{m}$.

Proof. Since each class $\mathcal{A}_{i}$ is a pencil of lines, it defines a point $P_{i}$, the base of the pencil. Consider two possible cases. 


\section{S. YUZVINSKY}

Case 1. The points $P_{i}$ are collinear, lying on a line $\ell$. Deleting $\ell$ from $\mathbb{P}^{2}$, we reduce this case to a 2-net in $\mathbb{C}^{2}$ each class of which consists of lines parallel to each other (cf. [DK74, $\left.\S 8.3\right]$ ). Choose an affine coordinate system so that the first class is formed by the lines $x=a_{i}$, the second one by $y=b_{i}$, and the third one by $x+y=c_{i}$, where $a_{i}, b_{i}, c_{i} \in \mathbb{C}$ and $i=1,2, \ldots, m$. We can choose the origin of the system so that the lines $x=0, y=0$, and $x+y=0$ are in the respective classes. Now we identify each class with a subset of $\mathbb{C}$ assigning to each line the right-hand side of its equation. Under this identification the resulting quasigroup (see the Introduction) becomes a subgroup of the additive group $\mathbb{C}$, which contradicts the finiteness of $N$.

Case 2. The points are not collinear. Choose a coordinate system such that $P_{1}=(0: 0: 1)$, $P_{2}=(0: 1: 0), P_{3}=(1: 0: 0)$. This allows us to write equations of lines in the classes as $a_{i} x-y=0, x-b_{i} z=0$, and $y-c_{i} z=0$ respectively, where $a_{i}, b_{i}, c_{i} \in \mathbb{C}^{*}$ and $i=1,2, \ldots, m$. Again we can normalize the equations of one line from each class to $x-y=0, x-z=0$, and $y-z=0$ respectively, and then identify classes with $\mathbb{C}^{*}$ assigning to a line the coefficient $a_{i}, b_{i}$, or $c_{i}$ respectively. We then obtain a quasigroup which is a subgroup of $\mathbb{C}^{*}$ whence isomorphic to $\mathbb{Z}_{m}$. Fixing a primitive $m$ th root of 1 as a generator and multiplying equations in each class, we obtain the result.

Remark 3.4. I have a little doubt that Proposition 3.3 is known. Section 8.3 of [DK74] is devoted to a similar topic. However, I could not find there the statement we need. The proof is easy and is included for convenience of the reader.

In the next section we will need the following corollary of Proposition 3.3.

Corollary 3.5. Let $N=(\mathcal{A}, \mathcal{X})$ be a $(3, m n)$-net $(m \geqslant 3)$ such that each class $\mathcal{A}_{i}$ is partitioned into $n$ blocks of size $m$, i.e. $\mathcal{A}_{i}=\bigcup_{j=1}^{n} \mathcal{A}_{i j}$, and for every pair $i, j$ there is a $k$ such that $\mathcal{A}_{1 i}, \mathcal{A}_{2 j}$, and $\mathcal{A}_{3 k}$ form three classes of a $(3, m)$-subnet $N_{i j}$ of $N$. Then if for every $i, j$ each class of $N_{i j}$ is a pencil, every class of $N$ is also a pencil.

Proof. Denote by $X_{i j}$ the base of the pencil $\mathcal{A}_{i j}$ and suppose the conclusion is false. Then without loss of generality we can assume that $X_{11} \neq X_{12}$. Consider the two nets $N_{11}$ and $N_{21}$. They have the same second class $\mathcal{A}_{21}$ and distinct first classes $\mathcal{A}_{11}$ and $\mathcal{A}_{12}$. By Proposition 3.3 there is a projective isomorphism $\phi$ of $\mathbb{P}^{2}$ fixing every line from $\mathcal{A}_{21}$ and mapping lines from $\mathcal{A}_{11}$ to lines from $\mathcal{A}_{12}$. Since $m \geqslant 3, \phi$ fixes every line passing through $X_{21}$, in particular the lines $l_{1}=\left(X_{21} X_{11}\right)$ and $l_{2}=\left(X_{21} X_{12}\right)$. (Here and below $(a b)$ denotes the line passing through points $a$ and $b$.) Besides $\phi\left(X_{11}\right)=\phi\left(X_{12}\right)$, we see that the points $X_{11}, X_{12}$, and $X_{21}$ are collinear.

Now there exists $k$ such that $\mathcal{A}_{3 k}$ is the third class in $N_{11}$. Substitute the class $\mathcal{A}_{3 k}$ for $\mathcal{A}_{21}$ and consider $N_{11}$ together with the $(3, m)$-subnet defined by $\mathcal{A}_{12}$ and $\mathcal{A}_{3 k}$. We see similarly to the above that $X_{11}, X_{12}$, and $X_{3 k}$ are collinear also. This implies that the bases $X_{11}, X_{21}$, and $X_{3 k}$ of the pencils of the net $N_{11}$ are collinear, which contradicts Proposition 3.3. The contradiction completes the proof.

Example 3.6. The only example of a 4-net in $\mathbb{P}^{2}$ known to the author is the Hessian configuration. The set of points for the net consists of the nine inflection points of a nonsingular plane cubic and the set of lines consists of the 12 lines each passing through three of these points. In this example $m=3$ and each of the four classes is in general position (i.e. its lines intersect at three distinct points).

No example of a 5-net in $\mathbb{P}^{2}$ is known to the author. 


\section{Realization of finite Abelian groups by NEts in $\mathbb{P}^{2}$}

\section{Realization of finite Abelian groups: first examples}

In the rest of this paper we will focus our attention on 3-nets. Let us recall the important feature of 3-nets from the Introduction. For a 3-net any pair $\left(l_{1}, l_{2}\right), l_{i} \in \mathcal{A}_{i}(i=1,2)$, defines a unique line $l_{3} \in \mathcal{A}_{3}$ passing through the point $l_{1} \cap l_{2}$. Thus we obtain a pairing $\mathcal{A}_{1} \times \mathcal{A}_{2} \rightarrow \mathcal{A}_{3}$ or, after some identification of each of three classes with a set $H$, a binary operation on $H$. This operation defines on $H$ a structure of a quasigroup or (under appropriate identification) even a loop (see [DK74]). If there exists an identification such that this loop is an Abelian group, then we say that this group is realized by the 3 -net.

We remark at this point that all known examples of 3-nets in $\mathbb{P}^{2}$ realize Abelian groups. So the rest of this paper is devoted to the question of which finite Abelian groups can be realized by a 3 -net (in $\mathbb{P}^{2}$ ).

First we prove a negative result.

Lemma 4.1. Suppose there are nine points $a_{1}, a_{2}, a_{3}, b_{1}, b_{2}, b_{3}, c_{1}, c_{2}, c_{3}$ such that the following triples are collinear: $\left\{a_{1}, b_{1}, c_{1}\right\},\left\{a_{1}, b_{2}, c_{2}\right\},\left\{a_{1}, b_{3}, c_{3}\right\},\left\{a_{2}, b_{1}, c_{3}\right\},\left\{a_{2}, b_{3}, c_{1}\right\},\left\{a_{3}, b_{2}, c_{3}\right\}$ and $\left\{a_{3}, b_{3}, c_{2}\right\}$. Then the lines $\left\{\left(a_{2} a_{3}\right),\left(b_{1} b_{2}\right),\left(c_{1} c_{2}\right)\right\}$ intersect at one point.

Proof. The triangles $b_{1} b_{2} c_{3}$ and $c_{1} c_{2} b_{3}$ form a Desargus configuration. More precisely, the lines passing through pairs of corresponding vertices intersect at $a_{1}$. Besides $\left(b_{1} c_{3}\right) \cup\left(c_{1} b_{3}\right)=\left\{a_{2}\right\}$ and $\left(b_{2} c_{3}\right) \cap\left(c_{2} b_{3}\right)=\left\{a_{3}\right\}$. Now the result follows from the second Desargus theorem.

TheOREM 4.2. The group $\mathbb{Z}_{2}^{3}$ cannot be realized.

Proof. Suppose that $\mathbb{Z}_{2}^{3}$ is realized by three sets of points $\left\{a_{i}\right\},\left\{b_{i}\right\}$ and $\left\{c_{i}\right\}$ where $i=1,2, \ldots, 8$. Let us consider the matrix $C$ of the pairing generated by the collinearity relation. For that we enumerate the rows of this matrix by $a_{i}$ and the columns by $b_{j}$. The $\left(a_{i}, b_{j}\right)$ entry is the point $c_{k}$ lying on the line $\left(a_{i} b_{j}\right)$. Without loss of generality we can assume that

$$
C=\left(\begin{array}{ll}
A & B \\
B & A
\end{array}\right)
$$

where

$$
A=\left(\begin{array}{llll}
c_{1} & c_{2} & c_{3} & c_{4} \\
c_{2} & c_{1} & c_{4} & c_{3} \\
c_{3} & c_{4} & c_{1} & c_{2} \\
c_{4} & c_{3} & c_{2} & c_{1}
\end{array}\right)
$$

and

$$
B=\left(\begin{array}{llll}
c_{5} & c_{6} & c_{7} & c_{8} \\
c_{6} & c_{5} & c_{8} & c_{7} \\
c_{7} & c_{8} & c_{5} & c_{6} \\
c_{8} & c_{7} & c_{6} & c_{5}
\end{array}\right)
$$

Using Lemma 4.1 for rows $a_{1}, a_{3}, a_{4}$ and columns $b_{1}, b_{2}, b_{4}$, we find that the lines $\left\{\left(a_{3} a_{4}\right),\left(b_{1} b_{2}\right)\right.$, $\left.\left(c_{1} c_{2}\right)\right\}$ intersect at a common point, say $x$. Using the lemma for rows $a_{1}, a_{5}, a_{6}$ and columns $b_{1}, b_{2}, b_{5}$, we find that $x \in\left(a_{5} a_{6}\right)$ and similarly $x \in\left(a_{7} a_{8}\right)$. Using the lemma again for rows $a_{5}, a_{7}, a_{8}$ and columns $b_{5}, b_{6}, b_{7}$, we have $x \in\left(b_{5} b_{6}\right) \cap\left(a_{1} a_{2}\right)$.

Now consider the quadrangle $a_{1} b_{1} b_{2} a_{2}$. We have $\left(a_{1} b_{1}\right) \cap\left(a_{2} b_{2}\right)=\left\{c_{1}\right\}$ and $\left(a_{1} b_{2}\right) \cap\left(a_{2} b_{1}\right)=\left\{c_{2}\right\}$. Thus by the diagonal theorem the points $x_{1}=\left(c_{1} c_{2}\right) \cap\left(a_{1} a_{2}\right)$ and $x_{2}=\left(c_{1} c_{2}\right) \cap\left(b_{1} b_{2}\right)$ form a harmonic pair for the pair $\left\{c_{1}, c_{2}\right\}$. By the inclusions in the previous paragraph, however, $x_{1}=x=x_{2}$. The contradiction completes the proof.

Using a similar (but simpler) argument, one can prove the following theorem. 


\section{S. YUZVINSKY}

TheOREm 4.3. There is no representation of $\mathbb{Z}_{2}^{2}$ with at least one class being a pencil.

Now we prove a positive result about the realization of finite Abelian groups by 3-nets.

Theorem 4.4. Let $H$ be a finite subgroup of a two-dimensional torus. Then there exists a 3-net in $\mathbb{P}^{2}$ realizing $H$.

Proof. Fix a nonsingular plane cubic curve $C$ and convert it to the two-dimensional torus choosing one of the flexes of $C$ as the neutral element. Then three points $p_{1}, p_{2}$, and $p_{3}$ from $C$ are collinear if and only if $p_{1}+p_{2}+p_{3}=0$. We can identify $H$ with a subset of $C$ and put $m=|H|$. Now choose $\alpha, \beta \in C / H$ such that $\alpha, \beta$, and $-\alpha-\beta$ are all distinct. The union of these three cosets is a set of $3 m$ points partitioned in three classes. It is clear that the lines in the projectively dual plane dual to these points form a 3 -net and this net realizes $H$.

\section{Algebraic nets}

In this section, we introduce the main notion of the paper and prove the main results. The following definition is a discrete analogue of the definition of algebraic webs (cf. [Bla55]).

Definition 5.1. Let $N=(\mathcal{A}, \mathcal{X})$ be a 3 -net in $\mathbb{P}^{2}$ and $N^{*}=\left(\mathcal{A}^{*}, \mathcal{X}^{*}\right)$ be the pair of dual sets of points and lines respectively in the dual plane $\mathbb{P}^{*}$. We say that $N$ is algebraic if there exists a cubic curve $\mathcal{C} \subset \mathbb{P}^{*}$ containing $\mathcal{A}^{*}$ in the set $\mathcal{C}^{0}$ of its regular points.

Notice that the cubic in the above definition is not assumed smooth nor even irreducible.

In order to start proving that some nets are algebraic, we need an auxiliary definition.

Definition 5.2. We call a subset of nine points from $\mathbb{P}^{2}$ complete if it is the complete intersection of two split cubics.

The famous property of complete sets necessary for our purposes is the Chasles theorem [EGH96]: if a cubic passes through eight arbitrary points of a complete set $T$, then it passes through all nine of them. Moreover, the cubics passing through a complete set form a pencil, a one-dimensional projective space spanned by the split cubics $\mathcal{C}_{1}$ and $\mathcal{C}_{2}$ from the above definition.

In the proofs of the next two results we will consider certain subsets $T \subset \mathcal{A}^{*}$ with the classes $\mathcal{A}_{i}^{*}$ identified to a set $H$ under some bijections. To distinguish elements from different classes, we write sets as $T=\left\{T_{1}\left|T_{2}\right| T_{3}\right\}$ where $T_{i} \subset H$ is identified with a subset of $\mathcal{A}_{i}^{*}$ (we will suppress other \{\}$)$. Also if $a_{i} \in H(i=1,2,3)$ we denote by $\left(a_{1}\left|a_{2}\right| a_{3}\right)$ the line from $\mathcal{X}^{*}$ passing through the respective points from the classes.

Theorem 5.3. Let $(\mathcal{A}, \mathcal{X})$ be a 3 -net representing a cyclic group $\mathbb{Z}_{m}$. Then this net is algebraic.

Proof. If $m=2,3$, the set $\mathcal{A}^{*}$ contains no more than nine points that all lie on the union of three lines away from their intersection points. The result follows.

In the rest of the proof we assume that $m>3$. The set $\mathcal{A}^{*}$ is partitioned in three classes $\mathcal{A}_{i}^{*}$ $(i=1,2,3)$ of cardinality $m$. Our method of proof is to find a sufficient supply of complete subsets of $\mathcal{A}^{*}$, three from each class, such that the split cubics from Definition 5.2 are unions of lines from $\mathcal{X}^{*}$.

Since the net realizes $\mathbb{Z}_{m}$ we can identify each class $\mathcal{A}_{i}^{*}$ with $\mathbb{Z}_{m}$ such that $a_{1}+a_{2}+a_{3}=0$ for $a_{i} \in \mathcal{A}_{i}^{*}(i=1,2,3)$ if and only if the points are collinear (and lie on a line from $\mathcal{X}^{*}$ ). Also we write elements of $\mathbb{Z}_{m}$ as integers defined modulo $m$.

To illustrate our notation and start the proof, consider the set $T_{0}=\{0,1,2|0,1,2|-1,-2,-3\}$. It is complete. Indeed for the two cubics $\mathcal{C}_{1}=(0|1|-1) \cup(1|2|-3) \cup(2|0|-2)$ and 


\section{Realization of finite Abelian groups by nets in $\mathbb{P}^{2}$}

$\mathcal{C}_{2}=(0|2|-2) \cup(1|0|-1) \cup(2|1|-3)$ we clearly have $\mathcal{C}_{1} \cap \mathcal{C}_{2}=T_{0}$. We denote by $\mathcal{C}$ the cubic from the pencil with the base $T_{0}$ containing also the point $3 \in \mathcal{A}_{1}$. It follows immediately from Bézout's theorem that $T_{0} \subset \mathcal{C}^{0}$.

Now we note that if $\left\{A_{1}\left|A_{2}\right| A_{3}\right\}$ is a complete set then $\left\{A_{1}+a_{1}\left|A_{2}+a_{2}\right| A_{3}-a_{1}-a_{2}\right\}$ is also complete. This gives us several complete sets obtained from $T_{0}: T_{k, l}=\{k, k+1, k+2|l, l+1, l+2|$ $-k-l-1,-k-l-2,-k-l-3\}$, where $k, l=0,1, \ldots, m-1$.

Now we introduce sets $T(s)$ of $3(s+1)$ points, $s=2,3, \ldots, m-1$, putting $T(s)=\{0, \ldots, s \mid$ $0, \ldots, s \mid 1, \ldots, s+1\}$. Since $T(m-1)=\mathcal{A}^{*}$ it suffices to prove that $T(s) \subset \mathcal{C}^{0}$ for all $s=2, \ldots, m-1$. We use finite induction on $s$.

First $T(2)=T_{0} \subset \mathcal{C}^{0}$ by construction. Suppose $s>2$ and $T(s-1) \subset \mathcal{C}^{0}$. The inductive step consists actually of three steps. First we notice that eight points of $T_{s-3,1}$ are on $\mathcal{C}^{0}$, whence the ninth one $\left(s+1\right.$ from $\left.\mathcal{A}_{3}\right)$ is on $\mathcal{C}^{0}$ also. Now eight points of $T_{s-2,0}$ are on $\mathcal{C}^{0}$, whence $\mathcal{C}^{0}$ also passes through $s$ from $\mathcal{A}_{3}$. Finally the same is true for $T_{0, s-2}$, which implies $T(s) \subset \mathcal{C}^{0}$. (The last step is redundant for $s=3$.)

Now we are ready to prove our main theorem about the algebraic character of nets realizing finite Abelian groups.

Theorem 5.4. Let $H$ be a finite Abelian group with at least one element of order greater than 9. Then every realization of $H$ by a 3 -net in $\mathbb{P}^{2}$ is algebraic.

Proof. It follows from the condition on $H$ that $H=\mathbb{Z}_{m} \oplus G$ where $m>9$ and $G$ is an Abelian group, say of order $d$. In particular, every class $\mathcal{A}_{i}$ can be partitioned into $d$ parts of size $m$ each, and this partition induces a partition of the net into $d^{2}$ subnets each representing the group $\mathbb{Z}_{m}$. From Theorem 5.3, for each of those subnets there exists a cubic in the dual plane containing all the $3 m$ points dual to the lines of the net. Clearly there is a linear ordering on these cubics such that every two subsequent cubics have at least $m$ points in common.

Now the proof branches out. First, suppose that at least one of these cubics is irreducible. Then since $m>9$ the cubics coincide with each other, which concludes the proof in this case.

Second, suppose that all the cubics are reducible and at least one has a line, say $L$, and an irreducible quadric, say $Q$, as its components. It follows from the collinearity pattern of the points that the only possible way in which they can be distributed among the components is such that the points from one class, say $\mathcal{A}_{1}^{*}$, lie on $L$ and others on $Q$. Similarly to the previous paragraph we deduce that the quadric $Q$ is the common component of all these cubics (using induction on the linear order and the inequality $m>6$ ). In order to prove that the line components also coincide, we construct more cubics. First, notice that any 9-tuple $T=\{a, b, c|a, b, c| a+b, a+c, b+c\}$ is complete for every subset $\{a, b, c\} \subset H$. Indeed $T$ is the intersection of the cubics $\mathcal{C}_{1}=$ $(a|b| a+b) \cup(c|a| a+c) \cup(b|c| b+c)$ and $\mathcal{C}_{2}=(b|a| a+b) \cup(a|c| a+c) \cup(c|b| b+c)$. Notice also that any cubic of the pencil generated by $\mathcal{C}_{1}$ and $\mathcal{C}_{2}$ has six points lying on $Q$, whence we can fix a cubic $\mathcal{C}(T)$ from this pencil having seven such points. Thus $\mathcal{C}(T)=Q \cup L(T)$ where $L(T)$ is its linear component.

Now fix two subnets representing $\mathbb{Z}_{m}$ lying on cubics with linear components $L_{1}$ and $L_{2}$ respectively. Line $L_{i}$ contains a subset $\mathcal{A}_{1 i} \subset \mathcal{A}_{1}$ of $m$ points. Choose a 9-tuple $T_{1}$ as in the previous paragraph with the extra condition $a, b \in \mathcal{A}_{11}$ and $c \in \mathcal{A}_{12}$. Choose also a 9-tuple $T_{2}$ corresponding to a subset $\left\{a, b^{\prime}, c\right\} \subset H$ with $b^{\prime} \in \mathcal{A}_{12}$. Then $L\left(T_{1}\right)$ and $L\left(T_{2}\right)$ have the points $a$ and $c$ from $\mathcal{A}_{1}$ in common and thence coincide. On the other hand, $L\left(T_{i}\right)$ has two points from $\mathcal{A}_{1}$ common with $L_{i}$, whence $L_{1}=L_{2}$. This implies that all the linear components of the cubics containing the subnets coincide and hence the cubics themselves coincide. 


\section{S. YUZVINSKY}

Finally suppose that all the cubics containing subnets split into a union of three lines each. This means that for the dual $(3, m)$-nets each class is a pencil. Then by Corollary 3.5 the whole initial net has this property, whence the dual net lies on the split cubic formed by the lines dual to the bases of the pencils. This completes the proof.

\section{Remark 5.5.}

i) Notice that the condition $m>6$ suffices for the conclusion of Theorem 5.4 in the case where a class of the net is a pencil. The case where all three classes are pencils needs only $m>2$. In fact, from Theorem 4.2, the only unknown case in the latter situation is for the group $\mathbb{Z}_{2}^{2}$.

ii) Notice also that the commutativity of $H$ was really used only in the second case where each cubic containing a subnet had two irreducible components.

When a 3-net is algebraic it realizes a subgroup of the group defined on the smooth points of the cubic by the collinearity relation. First we recall these groups.

If a cubic $\mathcal{C}$ is smooth, then this relation on $\mathcal{C}$ uniquely defines the third point $\mu(a, b) \in \mathcal{C}$ for any given $a, b \in \mathcal{C}$. Taking an arbitrary inflection point $p_{0} \in \mathcal{C}$ as 0 one gets inverses via $-a=\mu(0, a)$ and the binary operation defined by $a+b=-\mu(a, b)$. This operation gives an Abelian group isomorphic to a 2-torus. Three points $p, q, r \in \mathcal{C}$ are collinear if and only if $p+q+r=0$.

If a cubic $\mathcal{C}$ is not smooth, we denote as above by $\mathcal{C}^{0}$ the complement in $\mathcal{C}$ to the singular locus. The following results about the algebraic structures on $\mathcal{C}^{0}$ are probably very old, but I could not find them in the literature.

In the following proposition we always denote by $\mathbb{C}^{*}$ the multiplicative group on nonzero complex numbers and by $\mathbb{C}$ the additive group on all complex numbers.

\section{Proposition 5.6.}

1) Let $\mathcal{C}$ be an irreducible singular cubic. Define the operation ${ }^{*}$ on $\mathcal{C}^{0}$ in exactly the same way as was done on a smooth cubic. Then $\mathcal{C}^{0}$ is invariant with respect to ${ }^{*}$ and forms a group isomorphic to $\mathbb{C}^{*}$ or $\mathbb{C}$ depending on the singular point being a node or a cusp respectively.

2) Let $\mathcal{C}$ have as the components a quadric $Q$ and a line $L$. Then $\mathcal{C}^{0}$ is a disjoint union of $Q^{0}$ and $L^{0}$ that are the complements in $Q$ and $L$ respectively to their common points. The collinearity relation gives a pairing $Q^{0} \times Q^{0} \rightarrow L^{0}$. There exist isomorphisms of $Q^{0}$ and $L^{0}$ (as quasiaffine varieties) to either $\mathbb{C}^{*}$ or $\mathbb{C}$ depending on whether $|L \cap Q|=2$ or 1 respectively.

3) Finally let $\mathcal{C}$ be the union of three distinct lines $L_{1}, L_{2}$, and $L_{3}$. Denote by $L_{i}^{0}$ the complement in $L_{i}$ to the intersection points with the other two lines, i.e. $\mathcal{C}^{0}=\bigcup_{i} L_{i}^{0}$. Then the collinearity relation defines a pairing $L_{1}^{0} \times L_{2}^{0} \rightarrow L_{3}^{0}$. There exist isomorphisms of $L_{i}^{0}$ to either $\mathbb{C}^{*}$ or $\mathbb{C}$ depending on whether $\left|\bigcap_{i} L_{i}\right|=3$ or 1 respectively.

Proof. That $\mathcal{C}^{0}$ is invariant with respect to the operation * follows for all the cases from Bézout's theorem. The other claims are proved by choosing convenient parametrizations and, for part 1, the neutral element. We will describe the choices, leaving the straightforward checking to the reader.

1a) Suppose that $\mathcal{C}$ is irreducible and has a node. We can assume that its equation is $x^{3}+x^{2} z-$ $y^{2} z=0$. A well-known parametrization of $\mathcal{C}$ over $\mathbb{P}^{1}$ is given by $\left(u_{1}: u_{2}\right) \mapsto\left(u_{2}\left(u_{1}^{2}-u_{2}^{2}\right)\right.$ : $\left.u_{1}\left(u_{1}^{2}-u_{2}^{2}\right): u_{2}^{3}\right)$. This parametrization induces an isomorphism of $\mathbb{C}^{*} \cong \mathbb{P}^{1} \backslash\{(1: \pm 1)\}$ to $\mathcal{C}^{0}=\mathcal{C} \backslash\{(0: 0: 1)\}$. Putting $t=\left(u_{1}-u_{2}\right) /\left(u_{1}+u_{2}\right)$ we get this isomorphism in the form

$$
t \mapsto \phi(t)=\left(4 t(1-t): 4 t(1-t):(1-t)^{3}\right) \quad\left(t \in \mathbb{C}^{*}, \phi(t) \in \mathcal{C}^{0}\right) .
$$

Then $\phi$ sends the multiplication on $\mathbb{C}^{*}$ to the operation on $\mathcal{C}^{0}$ defined by the collinearity and the neutral element $\phi(1)=(0: 1: 0)$. 


\section{Realization of finite Abelian groups by nets in $\mathbb{P}^{2}$}

1b) Suppose that $\mathcal{C}$ is irreducible and has a cusp. The standard equation is $x^{3}-y^{2} z=0$ and an isomorphism $\phi: \mathbb{C} \rightarrow \mathcal{C}^{0}$ is given by $\phi(s)=\left(s: 1: s^{3}\right)$. This isomorphism sends the addition on $\mathbb{C}$ to the operation on $\mathcal{C}^{0}$ defined by the collinearity and the neutral element $\phi(0)=(0: 1: 0)$.

2a) Suppose that the irreducible components of $\mathcal{C}$ are a quadric $Q$ and a line $L$ and that $|Q \cap L|=2$. The standard equations are $x^{2}+y^{2}-z^{2}$ and $z=0$ respectively. Isomorphisms $\phi_{1}: \mathbb{C}^{*} \rightarrow$ $Q^{0}$ and $\phi_{2}: \mathbb{C}^{*} \rightarrow L^{0}$ are given by $\phi_{1}(s)=\left(1+s^{2}: i\left(1-s^{2}\right): 2 s\right)$ and $\phi_{2}(s)=(1-s: i(1+s): 0)$ respectively. These isomorphisms send the multiplication on $\mathbb{C}^{*}$ to the pairing defined by the collinearity.

2b) Suppose the irreducible components of $\mathcal{C}$ are like in the previous case but $L$ is tangent to $Q$. The standard equations are $x^{2}-y z=0$ and $z=0$. Isomorphisms $\mathbb{C} \rightarrow Q^{0}$ and $\mathbb{C} \rightarrow L^{0}$ are given by $s \mapsto\left(s: s^{2}:-1\right)$ and $s \mapsto(1: s: 0)$. They send the addition to the pairing defined by the collinearity.

3a) Suppose that the components of $\mathcal{C}$ are distinct lines $L_{i}(i=1,2,3)$ not all passing through a common point. They can be described by the equations $x=0, y=0$, and $z=0$ respectively. Isomorphisms $\mathbb{C}^{*} \rightarrow L_{i}$ are given by $s \mapsto(0: s: 1), s \mapsto(0: 1: s)$, and $s \mapsto(1 / s:-s: 0)$ respectively. They send the multiplication to the pairing defined by the collinearity.

3b) Finally suppose that the components of $\mathcal{C}$ are distinct lines $L_{i}$ passing through a point $p$. They can be given by the equations $x=0, y=0$, and $x-y=0$ respectively. Isomorphisms $\mathbb{C} \rightarrow L_{i}$ are given by $s \mapsto(0: 1: s), s \mapsto(1: 0: s)$, and $s \mapsto(1: 1: s)$. They send the addition to the needed pairing.

From Proposition 5.6 and Theorem 5.4 we have the following immediate corollary.

Corollary 5.7. Let $H$ be a finite Abelian group.

i) Suppose $H$ has an element of order $\geqslant 10$. Then $H$ can be realized by a 3-net in $\mathbb{P}^{2}$ if and only if $H$ has at most two invariant factors.

ii) Suppose that $H$ has an element of order $\geqslant 7$. Then $H$ can be realized by a 3 -net one class of which is a pencil if and only if $H$ is cyclic.

\section{Conjectures and open problems}

We conjecture that the conditions on the order of elements of $H$ in Corollary 5.7 are not necessary. From Theorem 4.4, we know that it is true in one direction.

Conjecture 6.1. If a finite group $H$ can be realized by a 3 -net in $\mathbb{P}^{2}$, then $H$ is a direct sum of at most two cyclic groups. If one class of the net is a pencil, then $H$ is cyclic.

Using the result for $\mathbb{Z}_{2}^{3}$, to settle this conjecture it suffices to prove or disprove that the groups $\mathbb{Z}_{p}^{3}$ are not realizable for $p=3,5,7$.

The results of the previous section lead naturally to the following problems.

Problem 1. Do there exist 4-nets in $\mathbb{P}^{2}$ nonisomorphic to the Hessian configuration?

Problem 2. Do there exist 5-nets in $\mathbb{P}^{2}$ ?

Problem 3. Does there exist a 3 -net in $\mathbb{P}^{2}$ not realizing an Abelian group?

Problem 4. Do there exist nonalgebraic 3-nets in $\mathbb{P}^{2}$ ?

These problems are related. For instance, the negative solution of Problem 4 would solve Problem 2 completely (and negatively) and make significant progress in Problem 1. 


\section{Realization of finite Abelian groups by nets in $\mathbb{P}^{2}$}

\section{REFERENCES}

Bla55 W. Blaschke, Einführung in die Geometrie der Waben (Birkhäuser, Basel, 1955).

DK74 J. Dénes and A. D. Keedwell, Latin squares and their applications (Academic Press, New York, 1974).

EGH96 D. Eisenbud, M. Green and J. Harris, Cayley-Bacharach theorems and conjectures, Bull. Amer. Math. Soc. 33 (1996), 295-324.

Kac90 V. Kac, Infinite dimensional Lie algebras (Cambridge University Press, Cambridge, 1990).

LY00 A. Libgober and S. Yuzvinsky, Cohomology of the Orlik-Solomon algebras and local systems, Compositio Math. 121 (2000), 337-361.

OT92 P. Orlik and H. Terao, Arrangements of hyperplanes (Springer, Berlin, 1992).

YO01 S. Yuzvinsky, Orlik-Solomon algebras in algebra and topology, Russian Math. Surveys 56 (2001), $87-166$.

Sergey Yuzvinsky yuz@math.uoregon.edu

Department of Mathematics, University of Oregon, Eugene, OR 97403, USA 